\title{
Characteristics and predictors of mortality on haemodialysis in Brazil: a cohort of 5,081 incident patients
}

\author{
Ana Beatriz Lesqueves Barra ${ }^{1,2}$, Ana Paula Roque-da-Silva' ${ }^{1}$, Maria Eugenia F. Canziani ${ }^{3}$, Jocemir R. Lugon ${ }^{1,4}$ and
} Jorge Paulo Strogoff-de-Matos ${ }^{1,4^{*}}$

\begin{abstract}
Background: Although Brazil has one of the largest populations on haemodialysis (HD) in the world, data regarding patients' characteristics and the variables associated with risk of death are scanty.

Methods: This is a retrospective analysis of all adult patients who initiated on maintenance HD at 23 dialysis centres in Brazil between 2012 and 2017. Patients were censored after 60 months of follow-up or at the end of 2019.

Results: A total of 5,081 patients were included in the analysis. The median age was 59 years, $59.4 \%$ were men, $37.5 \%$ had diabetes as the cause of kidney failure. Almost $70 \%$ had a central venous catheter (CVC) as the initial vascular access, about $60 \%$ started dialysis in the hospital, and fluid overload (FO) by bioimpedance assessment was seen in $45 \%$ of patients. The 60 -month survival rate was $51.4 \%$. In the Cox regression analysis, being older $(P<0.0001)$, starting dialysis in the hospital $(P=0.016)$, having diabetes as the cause of kidney failure $(P=0.001)$, high alkaline phosphatase $(P=0.005), C V C$ as first vascular access $(P=0.023)$, and FO $(P<0.0001)$ were associated with higher death risk, whereas higher body mass index $(P=0.015)$, haemoglobin $(P=0.004)$, transferrin saturation $(P=0.002)$, and serum albumin $(P<0.0001)$ were associated with better survival. The same variables, except initial $C V C$ use $(P=0.14)$, were associated with death risk in an analysis of subdistribution proportional hazards ratio including the competing outcomes.

Conclusions: The present study gives an overview of a large HD population in a developing country and identifies the main predictors of mortality, including some potentially modifiable ones, such as unplanned initiation of dialysis in the hospital and fluid overload.
\end{abstract}

Keywords: End-stage renal disease, Haemodialysis, Epidemiology, Survival analysis, Brazil

\section{Introduction}

The prevalence of kidney failure is increasing worldwide. Haemodialysis (HD) is the most common treatment method for this population. Brazil has one of the largest populations on HD in the world, and the number of

\footnotetext{
*Correspondence: strogoff@uol.com.br

${ }^{4}$ Nephrology Division, Department of Medicine, Universidade Federal

Fluminense, Av. Marquês do Paraná 303, 2 andar, Niterói, Rio de

Janeiro Zip Code 24033-900, Brazil

Full list of author information is available at the end of the article
}

patients has increased from nearly 87,000 to 133,000 over the last 10 years [1].

The life expectancy of patients undergoing HD remains dramatically shorter than in the general population, although a trend for improvement in the survival over the years has been recently documented in the USRDS [2] and the ERA-EDTA Registry [3]. According to 2020 USRDS Annual Report, 58\% of the patients on HD are alive after 3 years and only $41 \%$ after 5 years of therapy [2]. Of note, the mortality rate is substantially high in the first year after dialysis initiation, decreases markedly 
in the second year, and exhibits a steady increase over time [2, 4]. In Brazil, mortality data of the patients on HD are limited since the nationwide registry is a sample that comes from voluntary reports by the clinics. The latest reported data show an annual gross mortality rate of $19.5 \%[1]$.

Several factors have been associated with mortality of HD patients, including older age, presence of diabetes and cardiovascular disease, and some potentially modifiable factors such as the presence of a central venous catheter, hypervolemia, and anaemia [5, 6]. Moreover, differences in patient characteristics, environment factors and health care plans can potentially impact the outcome [7]. Overall, the knowledge about HD patients and factors influencing mortality come mainly from developed countries. This information, which is scarce in Brazil, could be a useful reference for further studies and healthcare policy. Thus, the present study aimed to evaluate the main predictors of mortality in a large HD population of a developing country.

\section{Methods}

This is a retrospective analysis of all incident HD patients from July 01, 2012 to June 30, 2017 in 23 Fresenius Medical Care dialysis centres in Brazil. The dialysis centres were distributed among 6 out of 27 states of Brazil (Rio de Janeiro, São Paulo, Minas Gerais, Bahia, Pernambuco, and Brasília - Federal District). All data were extracted from the European Clinical Dialysis Database $\left(\mathrm{EuCliD}^{\circledR}\right)$, a standardized electronic database used by the participating centres.

Patients under 18 years of age and those who underwent peritoneal dialysis or kidney transplantation before starting HD were excluded. Demographic, clinical and laboratory data on the admission to the clinic were used to define the baseline characteristics of the patients. At the onset of the maintenance haemodialysis, the patients had the body composition analysed by bioimpedance spectroscopy (BIS) - $\mathrm{BCM}^{\circledR}$, Fresenius Medical Care. They were classified as fluid overloaded if pre-dialysis excessive extracellular volume was higher than $13 \%$ for women and $15 \%$ for men [8].

Data were censored at 60 months of follow-up or on December 31, 2019. Data were also censored in cases of transfer to another dialysis centre, kidney transplant, migrating to peritoneal dialysis, recovery of renal function or abandonment of therapy. The primary outcome was all-cause mortality. This study was performed in accordance with the Declaration of Helsinki and was approved by the local ethics committee under the number CAAE 76623317.1.0000.5243. No written informed consent was requested due to the retrospective nature of the study.

\section{Statistical analysis}

Kolmogorov-Smirnov test was used to test for the distribution of variables. Continuous variables with normal distribution were expressed as mean \pm standard deviation or as median and interquartile range otherwise. Categorical variables were presented as frequencies. Comparisons between means were made using the unpaired $\mathrm{t}$ test or Mann-Whitney test and comparisons between frequencies were made using the chi-square test. The survival rate was calculated by Kaplan-Meier method and curves were compared by the Log-Rank test. The hazard ratios for death were estimated by Cox proportional hazards regression. Initially, univariate analysis was performed for each variable of interest and, subsequently, only those variables that showed $p$-values lower than 0.10 in the univariate assessment were included in the multivariate analysis. Additionally, similar analyses were done with adjustment for competing outcomes (kidney transplantation, migration to peritoneal dialysis, and kidney function recovery) using the subdistribution proportional hazards model described by Fine and Gray [9]. $P$-values $<0.05$ were considered statistically significant. All analyses were performed using SPSS version 18.0 for Windows (IBM $\odot$, Chicago, IL, USA), except the subdistribution hazard ratio analysis by the Fine-Gray method, which was performed using the freely available software $\mathrm{R}$ version 4.0.2.

\section{Results}

A total of 5,081 incident patients on HD in the period were included in this analysis. The baseline characteristics of the patients are in Table 1. The median age was 59 years and $59.4 \%$ were men. Diabetes, followed by hypertension, was the most common cause of kidney failure. Almost $70 \%$ of patients had a central venous catheter as the initial vascular access, and nearly $60 \%$ initiated dialysis in the hospital. More than half of the patients had the treatment funded by the public healthcare system. Patients who had the treatment funded by private insurance were older and with a higher prevalence of diabetes. Comparisons between demographic and clinical characteristics of patients with public and private healthcare insurances are shown in in the Supplementary Appendix (Table S1).

By the end of the 60 -month period, $33.3 \%$ of the patients followed had died, $24.0 \%$ remained on HD, $21.6 \%$ were transferred to other dialysis units, $10.6 \%$ had received a kidney transplant, $5.0 \%$ had migrated to peritoneal dialysis, $4.7 \%$ recovered from renal failure, and $0.8 \%$ had their follow-up lost, as shown in Fig. 1.

The survival rates for all patients at $3,12,24,36,48$ and 60 months of follow-up were 93.0, 82.6, 73.5, 65.1, 57.7 
Table 1 Baseline characteristics of patients $(n=5,081)$

\begin{tabular}{|c|c|}
\hline Gender male, n (\%) & $3,018(59.4)$ \\
\hline Age, years & $59(47-69)$ \\
\hline Non-white race/ethnicity, n (\%) & $2,877(56.6)$ \\
\hline \multicolumn{2}{|l|}{ Primary cause of kidney failure, $\mathrm{n}(\%)$} \\
\hline Diabetes & $1,906(37.5)$ \\
\hline Hypertension & $1,317(25.9)$ \\
\hline Glomerulonephritis & $547(10.8)$ \\
\hline Polycystic kidney disease & $193(3.8)$ \\
\hline Others & $357(7.0)$ \\
\hline Unknown & $761(15.0)$ \\
\hline Public healthcare insurance, $\mathrm{n}(\%)$ & $2,951(58.1)$ \\
\hline Early referral to nephrologist, n (\%) & $1,982(39.0)$ \\
\hline \multicolumn{2}{|l|}{ Place of first dialysis session } \\
\hline Hospital & $3,060(60.2)$ \\
\hline Dialysis centre & $1,321(26.0)$ \\
\hline No information & $700(13.8)$ \\
\hline \multicolumn{2}{|l|}{ Initial vascular access, n (\%) } \\
\hline Native arteriovenous fistula & $1,509(29.7)$ \\
\hline Graft & $35(0.7)$ \\
\hline Temporary catheter & $2,847(56.0)$ \\
\hline Tunnelled catheter & $690(13.6)$ \\
\hline Hepatitis B infection, n (\%) & $33(0.6)$ \\
\hline Hepatitis C infection, n (\%) & $136(2.7)$ \\
\hline HIV infection, n (\%) & $48(0.9)$ \\
\hline Erythropoietin use, n (\%) & $2,231(43.9)$ \\
\hline Haemoglobin, g/dL & $9.8(8.4-11.3)$ \\
\hline Transferrin saturation, \% & $24(17-34)$ \\
\hline Ferritin, ng/mL & $362(155-722)$ \\
\hline $\mathrm{BUN}, \mathrm{mg} / \mathrm{dL}$ & $54(43-69)$ \\
\hline Serum albumin, g/L & $36(33-40)$ \\
\hline Potassium, mEq/L & $5.0(4.5-5.7)$ \\
\hline Phosphorus, mg/dL & $4.6(3.7-5.7)$ \\
\hline Corrected calcium, mg/L & $9.0(8.6-9.5)$ \\
\hline Intact parathyroid hormone, $\mathrm{pg} / \mathrm{mL}$ & $262(125-509)$ \\
\hline Alkaline phosphatase, UI/L & $96(73-136)$ \\
\hline Pre-HD Systolic BP, mmHg & $142(129-156)$ \\
\hline Pre-HD Diastolic BP, mmHg & $79(70-85)$ \\
\hline Body mass index, $\mathrm{Kg} / \mathrm{m}^{2}$ & $23.7(21.0-27.1)$ \\
\hline \multicolumn{2}{|l|}{ Bioimpedance spectroscopy assessment } \\
\hline Lean mass, \% & $49.3(39.8-60.4)$ \\
\hline Fat mass, \% & $34.0(25.7-41.4)$ \\
\hline Excessive extracellular volume, \% & $12.9(4.8-21.1)$ \\
\hline Fluid overload, n (\%) & $1,721(45.4)$ \\
\hline
\end{tabular}

Values are expressed as frequency (\%) or median (interquartile range). $B U N$ blood urea nitrogen; $H D$ haemodialysis; $B P$ blood pressure

and 51.4\%, respectively (Fig. 2A). Survival rate was lower for diabetics than non-diabetics (44.5\% versus $56.3 \%$; $p$ $<0.0001$ ), Fig. 2B; Also, it was significantly lower for the older patients: $73.0,52.7,37.5$ and $16.2 \%$ for those $<50$ years-old, 50 to 64 years-old, 65 to 80 years-old and $\geq 80$ years-old, respectively (Fig. 2C). A total of 3.791 patients (74.6\%) had BIS assessment at the admission, with 1.721 (45.4\%) of them fluid overloaded. Survival rate was significantly lower among these patients, compared to those without fluid overload (46.6\% versus $65.2 \%$; $p<0.0001$ ), Fig. 2D.

In the initial Cox regression model analysis with no adjustment, female gender, higher age, diabetes as cause of kidney failure, higher alkaline phosphatase, starting dialysis in the hospital, having catheter as initial vascular access, and fluid overload in BIS assessment were associated with higher death risk, whereas early referral to nephrologist, having higher body mass index (BMI), higher haemoglobin, transferrin saturation (TSAT), serum albumin, blood urea nitrogen (BUN), phosphorus, intact parathyroid hormone (iPTH), diastolic blood pressure and lean mass in BIS assessment were associated with reduction in the death risk. After adjustment, including in the model only variables with $p$ values $<0.10$ in the univariate analysis, the following variables persisted associated with increased risk of death: age, diabetes, starting dialysis in the hospital, having catheter as initial vascular access, BMI, haemoglobin, TSAT, serum albumin, alkaline phosphatase, and fluid overload (Table 2).

In the univariate analysis of subdistribution hazard ratio (sHR) by the Fine-Gray model, including kidney transplantation, migration to peritoneal dialysis and kidney function recovery as competing outcomes, the variables associated with death risk were the same found in the unadjusted Cox regression analysis. All those variables plus corrected calcium $(p=0.067)$ were included in the adjusted subdistribution proportional hazards model. Again, the variables associated with death risk were the same found in the Cox regression model, except having catheter as initial vascular access $(p=0.14)$, Table 3 .

\section{Discussion}

This observational study involving more than 5,000 patients contributes to outlining the profile of the incident patients on HD in an emerging country, which has one of the largest dialysis population in the world and sheds light on the main risk factors associated with their long-term risk of death. This information can be particularly valuable for the local health policy of developing countries since the vast majority of available data is derived from developed nations.

The 5-year survival rate in our study was higher than that usually seen in developed countries, excepting Japan [4]. According to the ERA Registry Annual Report 2019 [3], the unadjusted 5-year survival rate of dialysis incident patients in the period 2010-2014 in Europe was 


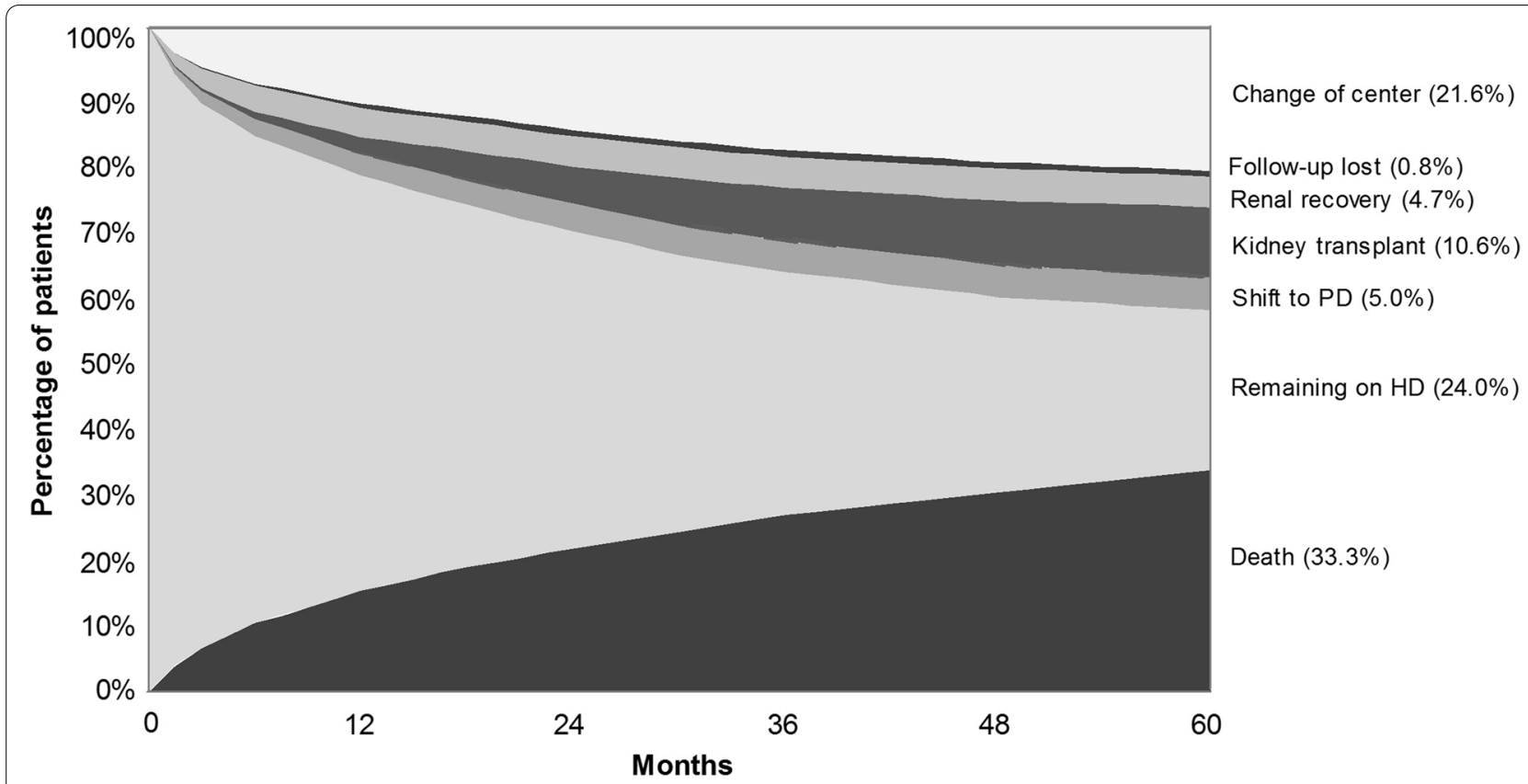

Fig. 1 Percentage of patients remaining on haemodialysis and the cumulative frequency of exit from the therapy, according to the cause, along the 60 -month period of follow-up

$42.3 \%$. Such difference could be explained by the markedly lower age and a much lower rate of kidney transplantation in our population, keeping younger and healthier patients on dialysis, consequently improving overall survival in the modality. As our incident dialysis population grows progressively older, an increase in mortality can be expected.

Indeed, we can see that the profile of the population incident on HD in Brazil is progressively catching up the ones of the developed countries accompanied by a trend of a negative impact in the survival rate. When comparing the population of the present study with the incident patients on HD between 2000 and 2004 in the same facilities chain in Brazil [10], the age at admission increased from 52 to 59 years, and diabetes as the underlying kidney disease rose from $20.4 \%$ to $37.5 \%$. Accordingly, the 5 -year survival rate decreased from $58.2 \%$ to $51.4 \%$. This could be justified not only by the differences of age and prevalence of diabetes between the two periods, but also by the inclusion of early deaths. In the present study, the mortality rate was $7 \%$ in the first 90 days on dialysis, which was proportionally much higher than throughout the remaining follow-up period, whereas in the previous study, patients who died in less than 90 days were excluded.

As expected, we found a strong association between both, older age and diabetes as the underlying kidney disease, with the risk of death. Likewise, we observed a significant increase in the risk of death for patients who started unplanned dialysis at a hospital. Unfortunately, most patients started under such circumstances, despite the wide recognition of that condition as a potentially modifiable risk factor [11], bringing to light the shortcomings of CKD management in the pre-dialysis phase by our health care system, either public or private.

Regarding laboratory variables on admission, higher serum albumin levels were associated with a lower death risk, which is a well-known association [12]. However, we cannot imply whether this would be a modifiable risk factor or just a marker of underlying clinical conditions not addressed in the analysis.

Higher alkaline phosphatase levels, but not iPTH, was associated with an increased risk of death. It is possible that serum alkaline phosphatase levels better reflect the bone mineral disorder, whereas it is known that $\mathrm{iPTH}$ levels in haemodialysis patients, in a wide range, varying from 2 to 9 times the upper limit of normality in the general population, have a weak correlation with histopathological findings on bone biopsy [13]. Similarly, a previous study showed the association between serum alkaline phosphatase levels and mortality risk in patients with advanced CKD transitioning to dialysis. Higher alkaline phosphatase levels over the last 6 months before initiation of dialysis were independently associated with increased post kidney failure mortality risk [14]. 

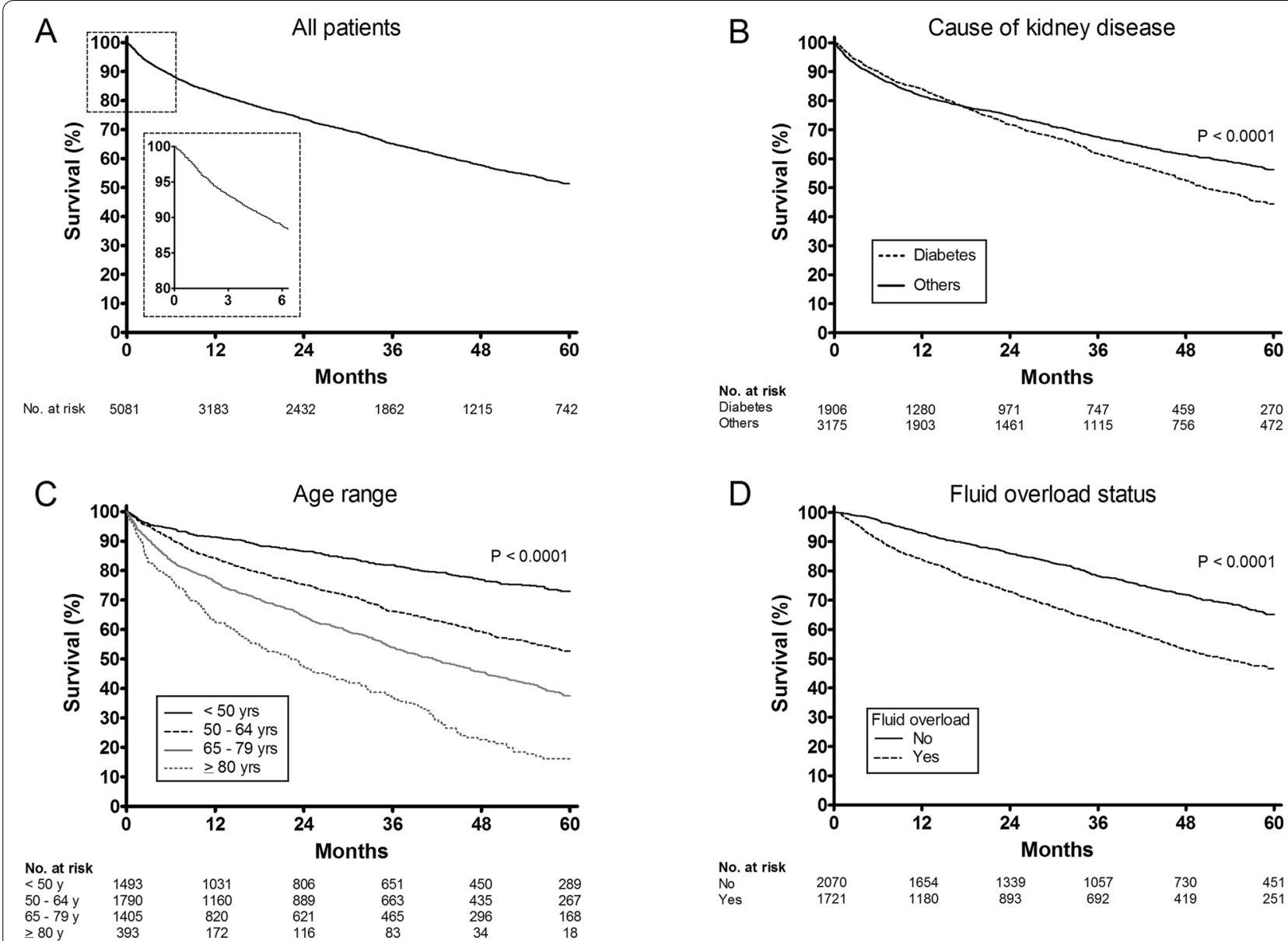

Fig. 2 60-month Kaplan-Meier survival curves, with starting of follow-up on the admission to the dialysis centre. A - All incident patients; B - According to the cause of kidney failure (diabetes vs. other causes); C - According to the age range; and $\mathbf{D}$ - According to fluid overload status assessed by bioimpedance spectroscopy at haemodialysis entrance

Almost half of the patients had no anaemia on admission and the vast majority did not present low haemoglobin levels that would demand blood transfusions. Most patients starting dialysis at a hospital may have had their anaemia treated and compensated for before discharge. In addition, outpatients were already undergoing care by nephrologists. Consistent with such interpretation, $44 \%$ of patients were already on EPO prescription at admission. We found an association between high haemoglobin levels at admission and low risk of death. It is interesting because the anaemia status is changed soon after HD start, with adequate EPO and iron replacement, targeting the same haemoglobin range for all patients along the follow-up period. The lower risk of death for patients with higher haemoglobin levels may reflect a better clinical condition not fully detected by the adjusted analysis model in the study or the need for higher EPO doses over the follow-up period in the patients who initially had lower haemoglobin levels.
It is interesting the association between TSAT $\geq 20 \%$ and the lower risk of death seen in the present study. Such association was found and well explored in a recent CKDopps study with pre-dialysis chronic kidney disease (CKD) patients [15]. However, whether low TSAT was just a marker of inflammation/malnutrition $[16,17]$ or iron replacement would be effective to improve outcomes is a question that could only be answered through interventional studies. According to the PIVOTAL study [18], a more vigorous iron replacement in patients on maintenance haemodialysis, but deliberately avoiding ferritin levels $\geq 700 \mathrm{ng} / \mathrm{mL}$ or TSAT $\geq 40 \%$, improved anaemia control, reduced erythropoiesis-stimulating agent doses and significantly reduced the risk of unfavourable clinical outcomes as compared to the control group, randomized to receive iron reactively when ferritin $<200 \mathrm{ng} / \mathrm{mL}$ or TSAT < $20 \%$. Thus, based on the findings of that study, we can 
Table 2 Cox regression for analysis of the risk of death in the 60-month period of follow-up, according to the baseline characteristics of the patients

\begin{tabular}{|c|c|c|c|c|}
\hline & Unadjusted HR (95\% Cl) & $P$-value & Adjusted HR (95\% Cl) & $P$-value \\
\hline Male & $0.81(0.74-0.90)$ & $<0.0001$ & $0.96(0.83-1.11)$ & 0.58 \\
\hline Age (years) & $1.04(1.03-1.04)$ & $<0.0001$ & $1.03(1.03-1.04)$ & $<0.0001$ \\
\hline Non-white race/ethnicity & $0.94(0.85-1.04)$ & 0.25 & - & - \\
\hline Diabetes & $1.23(1.12-1.36)$ & $<0.0001$ & $1.29(1.11-1.50)$ & 0.001 \\
\hline Public healthcare & $1.01(0.91-1.11)$ & 0.87 & - & - \\
\hline Early referral & $0.62(0.55-0.70)$ & $<0.0001$ & $0.88(0.74-1.05)$ & 0.16 \\
\hline First dialysis in hospital & $1.62(1.43-1.83)$ & $<0.0001$ & $1.29(1.05-1.58)$ & 0.016 \\
\hline Catheter & $2.01(1.79-2.26)$ & $<0.0001$ & $1.22(1.03-1.44)$ & 0.023 \\
\hline BMI $\left(\mathrm{Kg} / \mathrm{m}^{2}\right)$ & $0.97(0.96-0.98)$ & $<0.0001$ & $0.98(0.96-0.99)$ & 0.015 \\
\hline Haemoglobin (g/dL) & $0.87(0.85-0.90)$ & $<0.0001$ & $0.95(0.91-0.98)$ & 0.004 \\
\hline $\mathrm{TSAT} \geq 20 \%$ & $0.67(0.60-0.75)$ & $<0.0001$ & $0.80(0.69-0.92)$ & 0.002 \\
\hline Ferritin $\geq 200 \mathrm{ng} / \mathrm{mL}$ & $1.09(0.98-1.22)$ & 0.12 & - & - \\
\hline Erythropoietin use & $0.96(0.87-1.05)$ & 0.37 & - & - \\
\hline BUN (mg/dL) & $0.99(0.99-0.99)$ & $<0.0001$ & $1.00(0.99-1.00)$ & 0.13 \\
\hline Serum albumin (g/L) & $0.92(0.91-0.93)$ & $<0.0001$ & $0.96(0.95-0.98)$ & $<0.0001$ \\
\hline Potassium (mEq/L) & $0.98(0.93-1.03)$ & 0.48 & - & - \\
\hline Phosphorus (mg/dL) & $0.89(0.86-0.92)$ & $<0.0001$ & $1.00(0.95-1.06)$ & 0.92 \\
\hline Corrected calcium (mg/L) & $1.02(0.99-1.06)$ & 0.14 & - & - \\
\hline iPTH (per 100 pg/mL) & $0.95(0.94-0.96)$ & $<0.0001$ & $1.00(0.98-1.02)$ & 0.81 \\
\hline ALP (per 100 UI/L) & $1.09(1.06-1.12)$ & $<0.0001$ & $1.09(1.03-1.16)$ & 0.005 \\
\hline Pre-HD SBP (mmHg) & $1.00(0.99-1.00)$ & 0.34 & - & - \\
\hline Pre-HD DBP (mmHg) & $0.98(0.98-0.99)$ & $<0.0001$ & $1.00(0.99-1.01)$ & 0.89 \\
\hline Lean mass (\%) & $0.99(0.98-0.99)$ & $<0.0001$ & $1.00(0.99-1.00)$ & 0.33 \\
\hline Fat mass (\%) & $1.00(0.99-1.00)$ & 0.51 & - & - \\
\hline Fluid overload & $1.88(1.66-2.14)$ & $<0.0001$ & $1.47(1.27-1.71)$ & $<0.0001$ \\
\hline
\end{tabular}

$H R$ hazard ratio; $C I$ confidence interval; $B M I$ body mass index; TSAT transferrin saturation; $B U N$ blood urea nitrogen; $i P T H$ intact parathyroid hormone; $A L P$ alkaline phosphatase; $H D$ haemodialysis; SBP systolic blood pressure; DBP diastolic blood pressure

assume that intravenous iron replacement for our incident patients with TAST $<20 \%$ could have a favourable effect on outcomes.

Patients with a higher BMI were shown to have a lower risk of death, confirming a well-known association seen in previous studies. This would happen because in the haemodialysis population, with an unacceptably high mortality rate, the benefits of the obesityrelated nutritional reserve would probably outweigh the cardiovascular risks associated with the metabolic disorders, having such phenomenon been called the "obesity paradox" [19]. Interestingly, in the present study, neither the fat mass index nor the lean mass index measured by BIS were significantly associated with death risk.

The presence of FO in a single assessment on admission was the variable that was found to have the strongest association with the risk of death, even in such a long followup period. However, it is questionable whether FO would be directly associated with the cause of death or would be just a marker of other clinical conditions with poor prognosis, such as heart failure, lower residual urinary volume or hypoalbuminemia, contributing to the extracellular volume expansion. Even if such clinical conditions are behind FO, the way the patients are treated forward and how FO is corrected will probably impact the outcomes. Indeed, the long-term exposition to excessive extracellular volume is a much stronger predictor of death risk than the presence of FO at admission on HD, as shown by Zoccali et al [8]. We demonstrated in a previous study that even prevalent patients with $\mathrm{FO}$ while on conventional HD, after switching to short daily HD and subsequent correction of FO, had the same survival rate as those patients on short daily HD who were not with FO when they were still on conventional HD [20]. This finding corroborates once again that FO would be a modifiable risk factor and that its correction should be considered a priority in the clinical management.

Our study has several limitations. The first is the retrospective nature of the analysis. Second, the lack of 
Table 3 Subdistribution hazard ratio (sHR) using the Fine-Gray method for analysis of the risk of death in the 60-month period of follow-up, according to the baseline characteristics of the patients

\begin{tabular}{|c|c|c|c|c|}
\hline & Unadjusted sHR (95\% Cl) & $P$-value & Adjusted sHR $(95 \% \mathrm{Cl})$ & $P$-value \\
\hline Male & $0.83(0.76-0.92)$ & 0.0002 & $0.96(0.82-1.11)$ & 0.57 \\
\hline Age (years) & $1.04(1.03-1.04)$ & $<0.0001$ & $1.03(1.03-1.04)$ & $<0.0001$ \\
\hline Non-white race/ethnicity & $0.96(0.87-1.06)$ & 0.41 & - & - \\
\hline Diabetes & $1.32(1.20-1.45)$ & $<0.0001$ & $1.31(1.12-1.53)$ & 0.0006 \\
\hline Public healthcare & $1.08(0.98-1.19)$ & 0.14 & - & - \\
\hline Early referral & $0.72(0.65-0.81)$ & $<0.0001$ & $0.88(0.73-1.05)$ & 0.15 \\
\hline First dialysis in hospital & $1.58(1.40-1.79)$ & $<0.0001$ & $1.31(1.07-1.61)$ & 0.009 \\
\hline Catheter & $1.77(1.58-1.99)$ & $<0.0001$ & $1.13(0.96-1.34)$ & 0.14 \\
\hline $\mathrm{BMI}\left(\mathrm{Kg} / \mathrm{m}^{2}\right)$ & $0.97(0.96-0.98)$ & $<0.0001$ & $0.98(0.96-0.99)$ & 0.008 \\
\hline Haemoglobin (g/dL) & $0.88(0.86-0.91)$ & $<0.0001$ & $0.96(0.92-0.99)$ & 0.014 \\
\hline $\mathrm{TSAT} \geq 20 \%$ & $0.70(0.63-0.78)$ & $<0.0001$ & $0.81(0.70-0.94)$ & 0.005 \\
\hline Ferritin $\geq 200 \mathrm{ng} / \mathrm{mL}$ & $1.08(0.96-1.20)$ & 0.19 & - & - \\
\hline Erythropoietin use & $0.97(0.88-1.06)$ & 0.49 & - & - \\
\hline BUN (mg/dL) & $0.99(0.99-1.00)$ & $<0.0001$ & $1.00(0.99-1.00)$ & 0.22 \\
\hline Serum albumin (g/L) & $0.93(0.92-0.94)$ & $<0.0001$ & $0.97(0.95-0.98)$ & 0.0002 \\
\hline Potassium (mEq/L) & $1.01(0.96-1.06)$ & 0.69 & - & - \\
\hline Phosphorus (mg/dL) & $0.90(0.87-0.93)$ & $<0.0001$ & $1.00(0.95-1.06)$ & 0.99 \\
\hline Corrected calcium (mg/L) & $1.03(1.00-1.06)$ & 0.067 & $1.01(0.96-1.06)$ & 0.67 \\
\hline iPTH (per 100 pg/mL) & $0.96(0.95-0.98)$ & $<0.0001$ & $1.00(0.98-1.02)$ & 0.89 \\
\hline ALP (per 100 UI/L) & $1.09(1.06-1.12)$ & $<0.0001$ & $1.08(1.02-1.14)$ & 0.007 \\
\hline Pre-HD SBP (mmHg) & $1.00(1.00-1.00)$ & 0.67 & - & - \\
\hline Pre-HD DBP (mmHg) & $0.98(0.98-0.99)$ & $<0.0001$ & $1.00(0.99-1.01)$ & 0.96 \\
\hline Lean mass (\%) & $0.99(0.98-0.99)$ & $<0.0001$ & $1.00(0.99-1.00)$ & 0.17 \\
\hline Fat mass (\%) & $1.00(1.00-1.01)$ & 0.26 & - & - \\
\hline Fluid overload & $1.93(1.72-2.17)$ & $<0.0001$ & $1.50(1.29-1.74)$ & $<0.0001$ \\
\hline
\end{tabular}

$C l$ confidence interval; BMI body mass index; TSAT transferrin saturation; BUN blood urea nitrogen; $i P T H$ intact parathyroid hormone; $A L P$ alkaline phosphatase; $H D$ haemodialysis; SBP systolic blood pressure; DBP diastolic blood pressure

BIS assessment on about a quarter of patients. This was because the random lack of registration of BIS evaluation in the electronic database in some clinics, especially in the first years, and also due to a significant number of patients who indeed did not undergo BIS assessment, especially those who were readmitted or died early. Another limitation would be some lack of representativeness of the patients in this study compared to the haemodialysis population in Brazil as a whole. Almost half of the patients in this study were from facilities located in the state of Rio de Janeiro, where only about $10 \%$ of the Brazilian population lives, and $42 \%$ of them had their treatment funded by the private healthcare system, while the average in Brazil is about 20\% [1]. Anyway, the characteristics of patients funded by the public and private systems are presented separately in the supplementary material (Table S1). This study also has its strengths, such as the large number of patients, the long period of follow-up and the inclusion of variables, such as BIS assessment.

\section{Conclusion}

This study gives an overview of a large CKD population incident on maintenance haemodialysis in a developing country and provides information on factors, several of them potentially modifiable, such as unplanned initiation of dialysis in the hospital and fluid overload, which were found to be associated with the risk of death over a 5 -year period.

\section{Abbreviations}

BIS: Bioimpedance spectroscopy; CVC: Central venous catheter; ERA-EDTA: European Renal Association - European Dialysis and Transplant Association; FO: Fluid overload; HD: Haemodialysis; USRDS: United States Renal Data System.

\section{Supplementary Information}

The online version contains supplementary material available at https://doi. org/10.1186/s12882-022-02705-x.

Additional file 1. 


\section{Acknowledgements \\ Not applicable.}

\section{Authors' contributions}

A.B.L.B. was involved with data collection and organization, analysis of the data, and manuscript writing. A.P.R.S. was involved with data collection, organization, and analysis of the data. M.E.F.C. helped with study design, data interpretation, and manuscript writing. J.R.L. helped with study design, data interpretation, and manuscript writing. J.P.S.M. conceived the study and was involved with every step of the project. All authors have read and approved the manuscript.

\section{Funding}

This study was supported by Coordenação de Aperfeiçoamento de Pessoal de Nivel Superior (CAPES), Ministry of Education, Brazil.

\section{Availability of data and materials}

The datasets used and analysed during the current study are available from the corresponding author on reasonable request.

\section{Declarations}

\section{Ethics approval and consent to participate}

This study was performed in accordance with the Declaration of Helsinki. The project was approved by the research ethics committee of the Department of Medicine, Universidade Federal Fluminense, Niterói, RJ, Brazil. Approved Project Number: CAAE 76623317.10000.5243. No written informed consent was requested due to the retrospective nature of the study.

\section{Consent for publication}

Not applicable.

\section{Competing interests}

A.B.L.B. is a Fresenius Medical Care Brazil employee.

J.P.S.M and M.E.F.C. received consultancy fees from Fresenius Medical Care Brazil.

A.P.R.S and J.R.L declare no conflict of interest.

\section{Author details}

${ }^{1}$ Postgraduation Program in Medical Sciences, Universidade Federal Fluminense, Niterói, Rio de Janeiro, Brazil. ${ }^{2}$ Fresenius Medical Care Brazil, Rio de Janeiro, Brazil. ${ }^{3}$ Escola Paulista de Medicina, Universidade Federal de São Paulo, São Paulo, Brazil. ${ }^{4}$ Nephrology Division, Department of Medicine, Universidade Federal Fluminense, Av. Marquês do Paraná 303, 2 andar, Niterói, Rio de Janeiro Zip Code 24033-900, Brazil.

Received: 9 November 2021 Accepted: 10 February 2022

Published online: 23 February 2022

\section{References}

1. Neves PDMM, Sesso RCC, Thomé FS, Lugon JR, Nascimento MM. Brazilian Dialysis Census: analysis of data from the 2009-2018 decade. J Bras Nefrol. 2020:42:191-200.

2. United States Renal Data System. 2020 USRDS annual data report: Epidemiology of kidney disease in the United States. National Institutes of Health, National Institute of Diabetes and Digestive and Kidney Diseases, Bethesda, MD, 2020

3. ERA-EDTA Registry: ERA-EDTA Registry Annual Report 2019. Amsterdam UMC, location AMC, Department of Medical Informatics, Amsterdam, the Netherlands, 2021.

4. Robinson BM, Zhang J, Morgenstern $\mathrm{H}$, et al. Worldwide, mortality risk is high soon after initiation of hemodialysis. Kidney Int. 2014;85:158-65.

5. Miskulin D, Bragg-Gresham J, Gillespie BW, et al. Key comorbid conditions that are predictive of survival among hemodialysis patients. Clin J Am Soc Nephrol. 2009;4:1818-26.

6. Karaboyas A, Morgenstern H, Li Y, et al. Estimating the fraction of first-year hemodialysis deaths attributable to potentially modifiable risk factors: Results from the DOPPS. Clin Epidemiol. 2020:12:51-60.
7. Bello AK, Levin A, Lunney M, et al. Status of care for end stage kidney disease in countries and regions worldwide: international cross-sectional survey. BMJ. 2019;367:15873.

8. Zoccali C, Moissl U, Chazot C, et al. Chonic Fluid overload and mortality in ESRD. J Am Soc Nephrol. 2017;28:2491-7.

9. Fine JP, Gray RJ. A proportional hazards model for the subdistribution of a competing risk. J Am Stat Assoc. 1999;94:496-509.

10. Matos JP, Almeida JR, Guinsburg A, et al. Assessment of a five-year survival on hemodialysis in Brazil: a cohort of 3,082 incident patients. J Bras Nefrol. 2011;33:436-41.

11. Bradbury BD, Fissell RB, Albert JM, et al. Predictors of early mortality among incident US hemodialysis patients in the Dialysis Outcomes and Practice Patterns Study (DOPPS). Clin J Am Soc Nephrol. 2007;2:89-99.

12. Owen WF Jr, Lew NL, Liu Y, Lowrie EG, Lazarus JM. The urea reduction ratio and serum albumin concentration as predictors of mortality in patients undergoing hemodialysis. N Engl J Med. 1993;329:1001-6.

13. Barreto FC, Barreto FC, Barreto DV, et al. K/DOQI-recommended intact PTH levels do not prevent low-turnover bone disease in hemodialysis patients. Kidney Int. 2008;73:771-7.

14. Sumida K, Molnar MZ, Potukuchi PK, et al. Prognostic significance of pre-end-stage renal disease serum alkaline phosphatase for post-endstage renal disease mortality in late-stage chronic kidney disease patients transitioning to dialysis. Nephrol Dial Transplant. 2018;33:264-73.

15. Guedes M, Muenz DG, Zee J, Bieber B, et al. Serum biomarkers of iron stores are associated with increased risk of all-cause mortality and cardiovascular events in nondialysis CKD patients, with or without anemia. J Am Soc Nephrol. 2021;32:2020-30.

16. Kuragano T, Joki N, Hase H, et al. Low transferrin saturation (TSAT) and high ferritin levels are significant predictors for cerebrovascular and cardiovascular disease and death in maintenance hemodialysis patients. PLoS One. 2020;15:e0236277.

17. Sato M, Hanafusa N, Tsuchiya K, Kawaguchi H, Nitta K. Impact of transferrin saturation on all-cause mortality in patients on maintenance hemodialysis. Blood Purif. 2019;48:158-66.

18. Macdougall IC, White C, Anker SD, et al. Intravenous iron in patients undergoing maintenance hemodialysis. N Engl J Med. 2019;380:447-58.

19. Doshi M, Streja E, Rhee CM, et al. Examining the robustness of the obesity paradox in maintenance hemodialysis patients: a marginal structural model analysis. Nephrol Dial Transplant. 2016;31:1310-9.

20. Barra ABL, Roque-da-Silva AP, Vasconcellos MS, Lugon JR, Strogoff-deMatos JP. Association between extracellular volume control and survival in patients on short daily haemodialysis. BMC Nephrol. 2020;21:153.

\section{Publisher's Note}

Springer Nature remains neutral with regard to jurisdictional claims in published maps and institutional affiliations.

Ready to submit your research? Choose BMC and benefit from:

- fast, convenient online submission

- thorough peer review by experienced researchers in your field

- rapid publication on acceptance

- support for research data, including large and complex data types

- gold Open Access which fosters wider collaboration and increased citations

- maximum visibility for your research: over $100 \mathrm{M}$ website views per year

At BMC, research is always in progress.

Learn more biomedcentral.com/submissions 